\title{
$73(125 I)$
}

Influence of age on the permanence of subcutaneous autografts of the spleen in rabbits.

\section{By David Marine and O. T. Manley.}

[From the H. K. Cushing Laboratory of Experimental Medicine, Western Reserve University, Cleveland, Ohio.]

We have removed the spleen and auto transplanted in 49 rabbits, varying in age from 26 days to over two years.

The method consists of introducing a small fragment of spleen, roughly 2-3 $\mathrm{mm}$. in diameter, beneath the subcutaneous fascia of the abdomen, through a skin incision which is then closed by suture. These transplants have been subjected to direct examination at approximately monthly intervals to check their taking, growth or absorption. All have taken, except for three instances and these failures were due to infection. The most striking observation was the rapid growth of the grafts in the young rabbits from one to three months old and the lack of growth in the one- and two-year-old rabbits, some of which have undergone complete absorption in three months, as shown by histological examination. On the other hand, none of the transplants in rabbits less than five months old have shown any tendency to undergo absorption and histological examination shows regeneration of the major splenic elements into normal looking, encapsulated, highly vascular little spleens.

We have not been able to complete the series, with rabbits of known ages, between the period of sexual maturity (fifth month) and one year. The marked growth and activity of the transplants in young rabbits as compared with the lack of growth and tendency to absorption in old rabbits may be a part of the normal growth of the animal. In favor of this view is the fact that further growth of the transplants has not been observed after adolescence. There are no reports of differences in the systemic effects of splenectomy relative to age, although there is some evidence from histological studies that a blood-forming function is present in early life and absent in adults. It is suggested that the age differences noted in the growth and activity of spleen auto- 
grafts in addition to their probable relation to the normal growth of the animal as a whole may also be related to the loss of one of the spleen's functions in early life, through that function being assumed by another tissue.

The ease with which the spleen of young rabbits can be autotransplanted into the subcutaneous tissues might be utilized in the study of its reactions or in chemical examinations, where multiple or control spleens in accessible locations are needed.

\section{$74(1252)$}

Chemical evidence for the presence of glycogen-like polysaccharide in the liver blood of diabetic animals.

\section{By J. J. R. Macleod.}

[From the Western Reserve University, Cleveland, Ohio.]

Histological evidence for the presence in the hepatic capillaries of diabetic animals of material which stains by Best's carmine method in the same manner as the glycogen present in the liver cells (Huber and Macleod) has led us to investigate whether a polysaccharide could be separated by chemical means from the blood of the vena cava. In the first experiments of this nature (with G. E. Simpson), the blood of the vena cava was collected in excess of alcohol and the resulting precipitate treated with $\mathrm{KOH}$ in the usual manner. From the hydrolysis mixture a small amount of alcohol-precipitable material was secured, which after purification gave a violet color with iodine and exhibited reducing properties on hydrolysis. This material was sometimes isolated from the blood of normal animals (etherized), as well as from that of diabetic animals. In all cases, however, the yields were very small and uncertain, so that $I$ have recently changed the method of isolation. Instead of precipitating with alcohol as the first step, the blood is now received directly into saturated alkali. On account of the high cost of $\mathrm{KOH}$, a series of preliminary experiments were performed to see whether, after hydrolysis of the protein and heating with $\mathrm{NaOH}$, a solution would be obtained from which the glycogen could be quantitatively precipitated by alcohol. 